\title{
Erratum to: Effects of neuromodulation in a cortical network model of object working memory dominated by recurrent inhibition
}

\author{
Nicolas Brunel · Xiao-Jing Wang
}

Published online: 24 June 2014

(c) Springer Science+Business Media New York 2014

\section{Erratum to: J Comput Neurosci} DOI 10.1023/A:1011204814320

Correction for Effects of neuromodulation in a cortical network model of object working memory dominated by recurrent inhibition, by Nicolas Brunel and Xiao-Jing Wang, (Journal of Computational Neuroscience, issue 11, published in July-August 2001, pp.63-85)

The authors note that the equation for $\psi(v)$ (average gating variable of NMDA channels as a function of presynaptic rates, equation following equation $6, p .80$ ) is not exact, contrary to what is stated in the paper. This equation was derived using an expansion in central moments of the variable $x$ which appears in the system of differential equations for the NMDA gating variable. The equation for the central moments is correct up to 3rd order, but not at higher orders. Nonetheless, this equation gives a very good approximation of the true average gating variable for the range of firing rates explored in the paper, and therefore this error does not have any noticeable effect on the results of the paper.

The authors also note some of the equations in the appendix lack a term proportional to $V_{L}$ : Eq. (24) should be replaced by

$\langle V\rangle=\mu_{\mathrm{E}}+V_{L}-\left(V_{\mathrm{thr}}-V_{\text {reset }}\right) \nu_{\mathrm{E}} \tau_{\mathrm{E}}$

Eq. (26) should be replaced by

$\mu_{\mathrm{act}}=\frac{\left(T_{\mathrm{E}, \mathrm{ext}} v_{\mathrm{ext}}+T_{\mathrm{E}, \mathrm{AMPA}} n_{\mathrm{act}}+\rho_{1} N_{\mathrm{act}}\right) V_{\mathrm{E}}+\rho_{2} N_{\mathrm{act}}\langle V\rangle+T_{\mathrm{EI}} v_{\mathrm{I}} V_{\mathrm{I}}}{S_{\mathrm{act}}}-V_{L} \frac{\left(S_{a c t}-1\right)}{S_{a c t}}$

Eq. (28) should be replaced by:

$\mu_{+}=\frac{\left(T_{\mathrm{E}, \mathrm{ext}} v_{\mathrm{ext}}+T_{\mathrm{E}, \mathrm{AMPA}} n_{+}+\rho_{1} N_{+}\right) V_{\mathrm{E}}+\rho_{2} N_{+}\langle V\rangle+T_{\mathrm{EI}} v_{\mathrm{I}} V_{\mathrm{I}}}{S_{+}}-V_{L} \frac{\left(S_{+}-1\right)}{S_{+}}$

The online version of the original article can be found at http://dx.doi.org/10.1023/A:1011204814320.

\footnotetext{
N. Brunel $(\bowtie)$

Departments of Statistics and Neurobiology, The University

of Chicago, Chicago, IL 60637, USA

e-mail: nbrunel@guchicago.edu

X. -J. Wang

Center for Neural Science, New York University,

New York, NY 10012, USA

e-mail: xjwang@nyu.edu
} 
Eq. (30) should be replaced by:

$\mu_{0}=\frac{\left(T_{\mathrm{E}, \mathrm{ext}} \nu_{\mathrm{ext}}+T_{\mathrm{E}, \mathrm{AMPA}} \nu_{\mathrm{E}}+\rho_{1} N_{\mathrm{E}}\right) V_{\mathrm{E}}+\rho_{2} N_{E}\langle V\rangle+T_{\mathrm{EI}} \nu_{\mathrm{I}} V_{\mathrm{I}}}{S_{0}}-V_{L} \frac{\left(S_{0}-1\right)}{S_{0}}$

We thank David Andrieux and Francis Song for pointing these errors to us. 